\title{
L’application cotangente des surfaces de type général
}

\author{
Xavier Roulleau
}

Received: 2 September 2008 / Accepted: 20 February 2009 / Published online: 12 March 2009

(C) Springer Science+Business Media B.V. 2009

\begin{abstract}
We study surfaces of general type $S$ whose cotangent sheaf is generated by its global sections. We define a map called the cotangent map of $S$ that enables us to understand the obstructions to the ampleness of the cotangent sheaf of $S$. These obstructions are curves on $S$ that we call "non-ample". We classify the surfaces with an infinite number of non-ample curves and we partly classify the non-ample curves.
\end{abstract}

Keywords Ample cotangent bundle $\cdot$ Cotangent map

Mathematics Subject Classification (2000) $\quad 14 J 29$

\section{Introduction}

On étudie dans le présent article l'amplitude du fibré cotangent des surfaces de type général quand ce fibré cotangent est engendré par sections globales.

Les propriétés des variétés à fibré cotangent ample sont discutées en [4]. A l'aide d'une construction due à Bogomolov, Conduché et Palmieri [3] ont montré que les ratios $\frac{c_{1}^{2}}{c_{2}}$ des nombres de Chern de telles surfaces forment un sous-ensemble dense dans l'intervalle [1, 2]. Une lecture attentive de leur papier et l'utilisation du théorème des sections hyperplanes de Lefschetz permettent de raffiner leur résultat comme suit:

Pour tout entier $q \geq 12$, la fermeture des ratio de Chern des surfaces d'irrégularité $q$, à fibré cotangent ample et engendré par ses sections globales, contient un intervalle non-vide $I_{q} \subset[1,2]$. La réunion des intervalles croissants $I_{q}$ est l'intervalle [1,2].

Plutôt que de construire des surfaces dont le fibré cotangent est ample, on propose d'étudier les obstruction à son amplitude. Ces obstructions bien comprise, on peut alors espérer caractériser les surfaces à fibré cotangent ample.

X. Roulleau $(\varangle)$

Graduate School of Mathematical Sciences, University of Tokyo, 3-8-1 Komaba, Meguro,

153-8914 Tokyo, Japan

e-mail: roulleau@ms.u-tokyo.ac.jp 
Rappelons d'abord la définition d'amplitude d'un fibré vectoriel $\mathcal{E}$ sur une variété $X$, telle qu'introduite par Hartshorne [9]:

Notons $\mathbb{P}\left(\mathcal{E}^{*}\right)$ le projectivisé du dual de $\mathcal{E}, \pi: \mathbb{P}\left(\mathcal{E}^{*}\right) \rightarrow X$ la projection et $\mathcal{O}_{\mathbb{P}\left(\mathcal{E}^{*}\right)}(1)$ le fibré inversible tautologique tel que:

$$
\pi_{*} \mathcal{O}_{\mathbb{P}\left(\mathcal{E}^{*}\right)}(1)=\mathcal{E} .
$$

Définition 1.1 Le fibré $\mathcal{E}$ est dit ample si $\mathcal{O}_{\mathbb{P}\left(\mathcal{E}^{*}\right)}(1)$ est ample.

Quand le fibré vectoriel $\mathcal{E}$ est engendré par l'espace de ses sections globales, on dispose du critère d'amplitude suivant, dû à Gieseker [5]:

Proposition 1.2 (Gieseker) Le fibré $\mathcal{E}$ est ample si et seulement si pour toute courbe $C \hookrightarrow X$, le fibré $\mathcal{E} \otimes \mathcal{O}_{C}$ n'a pas de quotient isomorphe à $\mathcal{O}_{C}$.

Pour aborder la question de l'amplitude du fibré cotangent, nous sommes amenés à poser l'hypothèse suivante sur la surface considérée:

Hypothèse 1.3 Nous travaillerons dans ce qui suit avec une surface $S$ définie sur $\mathbb{C}$, lisse, de type général, dont le fibré cotangent $\Omega_{S}$ est engendré par l'espace de ses sections globales $H^{o}\left(\Omega_{S}\right)$ et d'irrégularité $q=\operatorname{dim} H^{o}\left(\Omega_{S}\right)$ vérifiant $q>3$.

L'article [3] de Conduché et Palmieri montre que de telles surfaces existent abondamment. Notons $T_{S}=\Omega_{S}^{*}$ le fibré tangent, $\pi: \mathbb{P}\left(T_{S}\right) \rightarrow S$ la projection et $\mathcal{O}_{\mathbb{P}\left(T_{S}\right)}$ (1) le fibré tautologique. On dispose d'une identification naturelle:

$$
H^{o}\left(\mathbb{P}\left(T_{S}\right), \mathcal{O}_{\mathbb{P}\left(T_{S}\right)}(1)\right) \simeq H^{o}\left(\Omega_{S}\right) .
$$

Définition 1.4 Par hypothèse, le morphisme naturel $H^{o}\left(\Omega_{S}\right) \otimes \mathcal{O}_{S} \rightarrow \Omega_{S}$ est surjectif, le morphisme $H^{o}\left(\Omega_{S}\right) \otimes \mathcal{O}_{\mathbb{P}\left(T_{S}\right)} \rightarrow \mathcal{O}_{\mathbb{P}\left(T_{S}\right)}(1)$ est donc également surjectif et définit un morphisme:

$$
\psi: \mathbb{P}\left(T_{S}\right) \rightarrow \mathbb{P}\left(H^{o}\left(\Omega_{S}\right)^{*}\right)=\mathbb{P}^{q-1}
$$

appelé l'application cotangente de la surface.

Ce morphisme et la question de l'amplidude du fibré cotangent sont les principaux objets d'étude du présent article. En vue de la proposition 1.2, on pose la définition suivante

Définition 1.5 Une courbe $C \hookrightarrow S$ est dite non-ample si et seulement si le fibré $\Omega_{S} \otimes \mathcal{O}_{C}$ possède un quotient isomorphe à $\mathcal{O}_{C}$.

Les courbes non-amples constituent donc l'obstruction à l'amplitude du fibré cotangent ; nous en étudions les propriétés. L'application cotangente permet de traduire les propriétés algébriques du fibré cotangent par les propriétés géométriques de l'image de $\psi$. Ainsi une courbe $C \hookrightarrow S$ est non-ample si et seulement si il existe une section $t: C \hookrightarrow \mathbb{P}\left(T_{S}\right)$ contractée en un point $p$ par $\psi$. En ce cas, l'image de $\pi^{*} C$ par $\psi$ est un cône de sommet $p$.

Il est donc légitime de poser la définition suivante:

Définition 1.6 Un point $p$ de $\mathbb{P}^{q-1}$ est dit exceptionnel si la fibre de $\psi$ en $p$ est de dimension $>0$.

Nous noterons $\Delta$ le lieu des points exceptionnels. Un problème naturel est d'étudier $\Delta$ et de caractériser les surfaces pour lesquelles ce fermé est de dimension strictement positive.

Résumons les résultats obtenus en vue de ces problèmes: 
Proposition 1.7 L'image de l'application cotangente est de dimension 3.

Soit $p$ un point de $\mathbb{P}^{q-1}$. La fibre $\psi^{-1}(p)$ est de dimension au plus 1 .

Le lieu des points exceptionnels est de dimension au plus 1 .

Nous établissons ensuite une borne sur le degré de l'application cotangente et sur le degré de son image en fonction des nombres de Chern $c_{1}^{2}, c_{2}$ de la surface.

Soit $C \hookrightarrow S$ une courbe, on appellera suite cotangente de $C$ la suite exacte:

$$
0 \rightarrow \mathcal{O}_{C}(-C) \rightarrow \Omega_{S} \otimes \mathcal{O}_{C} \rightarrow \Omega_{C} \rightarrow 0 .
$$

Le théorème suivant classifie partiellement les courbes non-amples de la surface:

Théorème 1.8 Soit $C$ une courbe de la surface $S$, alors:

(a) La courbe $C$ est non-ample et vérifie $C^{2}<0$ si et seulement si $C$ est lisse de genre 1.

(b) La courbe $C$ est non-ample et vérifie $C^{2}=0$ si et seulement si $C$ est lisse de genre $>1$ et la suite cotangente est scindée.

Ce théorème s'obtient à partir d'un critère de lissité de Lipman [10]. La propriété (a) nous semble un résultat particulièrement intéressant : étant donné une courbe $C$ vérifiant $C^{2}<0$ sur une surface lisse $X$, il est facile de construire une fibré vectoriel $\mathcal{E}$ sur $X$ engendré par ses sections globales et tel que $C \hookrightarrow S$ soit la seule courbe pour laquelle $\mathcal{E} \otimes \mathcal{O}_{C}$ admette un quotient trivial. Ceci illustre de nouveau les proprietés particulières que possède le fibré cotangent d'une surface.

Rappelons qu'une surface fibrée $f: S \rightarrow B$ est dite isotriviale si ses fibres lisses sont isomorphes entre-elles. Donnons des exemples de courbes non-amples sur des surfaces:

\section{Proposition 1.9}

(a) Les fibres lisses d'une fibration de S sont non-amples si et seulement si la fibration est isotriviale.

(b) Soit $S=C^{(2)}$ le produit symétrique d'une courbe $C$ de genre $>3$ et non-hyperelliptique. Cette surface vérifie les hypothèses 1.3 et contient une infinité de courbes non-amples $C$ vérifiant $C^{2}=1$. L'image de l'application cotangente est la variété des sécantes d'une courbe.

La partie (a) résulte d'un critère dû à Martin-Deschamps [11].

On dispose ainsi d'exemples de surfaces ayant une infinité de courbes non-amples. Nous classifions ces surfaces dans le théorème suivant:

Théorème 1.10 Soit $S$ une surface possédant une infinité de courbes non-amples et telle que le lieu exceptionnel $\Delta$ ne soit pas une droite de $\mathbb{P}^{q-1}$. L'image de l'application cotangente vérifie alors l'une des deux propriétés suivantes:

(a) La surface $S$ est une surface fibrée isotriviale dont les fibres sont les courbes non-amples. Le lieu des points exceptionnels $\Delta$ est formé de deux courbes et l'image de l'application cotangente est la variété développée par les sécantes de ces deux courbes.

(b) L'image de l'application cotangente est la variété des sécantes d'une courbe. Une courbe non-ample $C \hookrightarrow S$ vérifie en ce cas: $C^{2}>0$. Il existe un morphisme fini de $S$ dans le produit symetrique $B^{(2)}$ d'une courbe $B$. 
Ce théorème s'obtient en étudiant l'image inverse d'un fermé de dimension 1 de $\Delta$. Je remercie vivement Igor Reider qui m'a proposé ce thème de travail.

Une partie de cet article a été rédigée au Max-Planck Institute de Bonn.

\section{Etude de l'application cotangente.}

2.1 Les définitions de l'application cotangente.

Rappelons quelques proprietés partagées par une surface $S, A$ sa variété d'Albanese, $\vartheta: S \rightarrow A$ un morphisme d'Albanese et $\Omega_{S}$ son fibré cotangent:

Lemme 2.1 ([7] p. 331). La différentielle $d \vartheta: T_{S} \rightarrow \vartheta^{*} T_{A}=H^{o}\left(\Omega_{S}\right)^{*} \otimes \mathcal{O}_{S}$ du morphisme $\vartheta$ est le dual du morphisme d'évaluation $H^{o}\left(\Omega_{S}\right) \otimes \mathcal{O}_{S} \rightarrow \Omega_{S}$.

Notons $p_{r}: S \times H^{o}\left(\Omega_{S}\right)^{*} \rightarrow H^{o}\left(\Omega_{S}\right)^{*}$ la projection sur le second facteur. Par définition, le morphisme $\vartheta$ est une immersion locale si le morphisme:

$$
p_{r} \circ d \vartheta_{s}: T_{S, s} \rightarrow H^{o}\left(\Omega_{S}\right)^{*}
$$

est injectif en tout point $s$ de $S$. Le lemme 2.1 permet ainsi une interprétation géométrique de la condition 1.3:

Corollaire 2.2 Le morphisme d'Albanese d'une surface est une immersion locale si et seulement si son fibré cotangent est engendré par ses sections globales.

Notation 2.3 Maintenant et pour le reste de cet article, $S$ est une surface vérifiant les hypothèses 1.3 et on conservera les notations de l'introduction.

Le corollaire suivant est une conséquence immédiate du lemme 2.1 et permet une interprétation plus géométrique de l'application cotangente:

Corollaire 2.4 L'application cotangente de S est le projectivisé du morphisme:

$$
p_{r} \circ d \vartheta: T_{S} \rightarrow H^{o}\left(\Omega_{S}\right)^{*} .
$$

L'application cotangente est donc le morphisme qui à un point de la surface et à une direction tangente associe la direction tangente dans la variété d'Albanese.

Regardons maintenant les propriétés de la restriction de l'application cotangente en la fibre $\pi^{-1}(s)$ d'un point $s$. La fibre en $s$ de la projection $\pi$ est la courbe $\mathbb{P}\left(T_{S, s}\right) \simeq \mathbb{P}^{1}$ et la restriction de $\mathcal{O}_{\mathbb{P}\left(T_{S}\right)}(1)$ à cette courbe est le fibré de degré 1 , donc:

Lemme 2.5 La restriction de l'application cotangente à la fibre $\pi^{-1}(s)$ (s point de $S$ ) est un plongement et son image est une droite de $\mathbb{P}^{q-1}$.

Notation 2.6 Pour un point s de $S$, on notera $L_{S} \hookrightarrow \mathbb{P}^{q-1}$ la droite image de la fibre $\pi^{-1}(s)$ par $\psi$.

L'image de l'application cotangente est donc la réunion des droites $L_{S}(s$ point de $S$ ). Notons $G(2, q)$ la grassmannienne des sous-espaces vectoriels de dimension 2 de $H^{o}\left(\Omega_{S}\right)^{*}$. Cette grassmannienne paramètre également les droites de l'espace projectif $\mathbb{P}^{q-1}$. 
Définition 2.7 Le morphisme de Gauss:

$$
\mathcal{G}: S \rightarrow G(2, q)
$$

de la surface $S$ est défini par la surjection $H^{o}\left(\Omega_{S}\right) \otimes \mathcal{O}_{S} \rightarrow \Omega_{S}$.

Par construction, le point $\mathcal{G}(s)$ représente la droite projective $L_{s}$, ou encore:

Corollaire 2.8 Le morphisme de Gauss est le morphisme qui à un point s de la surface associe le point de $G\left(2, H^{o}\left(\Omega_{S}\right)^{*}\right)$ représentant le plan:

$$
p_{r} \circ d \vartheta_{s}\left(T_{S, s}\right) \subset H^{o}\left(\Omega_{S}\right)^{*} .
$$

Le corollaire 2 de [14] montre que:

Lemme 2.9 (Ran) Le morphisme de Gauss est fini sur son image.

Soit $U$ le fibré universel de $G(2, q)$ et $\mathbb{P}(U)$ le projectivisé de $U$. La situation étudiée est donc la suivante:

$$
\begin{array}{ccc}
\mathbb{P}\left(T_{S}\right) & \stackrel{\widetilde{\psi}}{\rightarrow} \mathbb{P}(U) \stackrel{\pi_{1}}{\rightarrow} \mathbb{P}^{q-1} \\
\pi \downarrow & \downarrow \pi_{2} \\
S & \stackrel{\mathcal{G}}{\rightarrow} G(2, q)
\end{array}
$$

où $\pi_{1}, \pi_{2}$ sont les projections naturelles et le morphisme $\widetilde{\psi}$ vérifie $\pi_{1} \circ \widetilde{\psi}=\psi$.

2.2 Dimension des fibres et de l'image de l'application cotangente.

Cette section porte sur les fibres de l'application cotangente et la dimension de son image. Nous établissons que ces fibres sont de dimension au plus 1 et qu'il y a au plus une famille de dimension 1 de fibres $\psi^{-1}(p)$ de dimension 1 . Nous montrons ensuite que l'image de l'application cotangente est de dimension 3.

Lemme 2.10 Soit $p$ un point de $\mathbb{P}^{q-1}$. Le morphisme $\pi$ est injectif sur les points de la fibre $\psi^{-1}(p)$.

Démonstration Soit $s$ un point de $S$. Si l'intersection de $\pi^{-1}(s)$ et de $\psi^{-1}(p)$ est non vide, cette intersection est nécessairement un point car $\psi$ est un plongement sur la fibre $\pi^{-1}(s)$ (cf. lemme 2.5).

Notons $F$ l'image de l'application cotangente. Si $p$ est un point de $\mathbb{P}^{q-1}$, nous noterons $D_{p}$ l'image de la fibre $\psi^{-1}(p)$ par $\pi$ : le fermé sous-jacent est formé des points $s$ de la surface tels que la droite $L_{s}$ passe par $p$.

Proposition 2.11 Pour tout point $p$ de $\mathbb{P}^{q-1}$, la fibre $\psi^{-1}(p)$ et le schéma $D_{p}$ sont de dimension au plus 1 .

Démonstration L'application cotangente $\psi$ n'est pas constante donc pour tout point $p$ de $\mathbb{P}^{q-1}$, la dimension de $\psi^{-1}(p)$ est inférieure ou égale à 2.

Supposons que la fibre de $\psi$ en un point $p$ soit de dimension 2. Soit $S^{\prime}$ le schéma réduit associé à la fibre $\psi^{-1}(p)$. Par le lemme 2.10, la restriction $\pi_{\mid S^{\prime}}$ du morphisme $\pi$ à $S^{\prime}$ est injective sur les points de $S^{\prime}$. Puisque $\pi$ est propre, le morphisme:

$$
\pi_{\mid S^{\prime}}: S^{\prime} \rightarrow S
$$


est surjectif. Le morphisme $\pi_{\mid S^{\prime}}$ est bijectif, séparable dans la variété normale $S$ : c'est un isomorphisme (cf. [12] remarque 6.21). Il existe donc un morphisme $t: S \rightarrow \mathbb{P}\left(T_{S}\right)$ tel que $\pi \circ t: S \rightarrow S$ soit l'identité. A ce morphisme $t$ correspond un quotient:

$$
\Omega_{S} \rightarrow \mathcal{L}
$$

où $\mathcal{L}$ est le fibré inversible qui vérifie $t^{*} \mathcal{O}_{\mathbb{P}\left(T_{S}\right)}(1)=\mathcal{L}$ ( [8] chapitre II proposition 7.12). Le fibré $\mathcal{O}_{\mathbb{P} q-1}(1)$ est trivial au point $p$ et, puisque $\psi \circ t$ contracte $S$ au point $p$, le fibré:

$$
t^{*} \psi^{*} \mathcal{O}_{\mathbb{P} q-1}(1)=\mathcal{L}
$$

est trivial. Ainsi $\Omega_{S}$ possède un quotient d'image un fibré inversible trivial. Puisque $\Omega_{S}$ est engendré par $H^{0}\left(\Omega_{S}\right)$, ce quotient a une section et $\Omega_{S}=\mathcal{O}_{S} \oplus \mathcal{O}_{S}(K)$ ( $K$ diviseur canonique). Cela est impossible car nous avons supposé $S$ de type général. La fibre de $\psi$ en un point est donc de dimension au plus 1 .

Pour tout point $p$ de $\mathbb{P}^{q-1}$, l'image de la fibre $\psi^{-1}(p)$ par $\pi$ est donc également de dimension inférieure ou égale à 1 .

Soit $p$ un point de $\mathbb{P}^{q-1}$ tel que $D_{p}=\pi\left(\psi^{-1}(p)\right)$ soit de dimension 1 . Considérons $D$ une composante irréductible de dimension 1 de $D_{p}$ munie de sa structure réduite, alors :

Proposition 2.12 L'image par l'application cotangente de la surface réglée $\pi^{-1}(D)$ est un cône de sommet $p$.

Démonstration L'application $\psi$ est injective sur les fibres de $\pi$, le fermé irréductible $\psi\left(\pi^{-1}\right.$ $(D))$ est donc au moins de dimension 1.

Si $\psi\left(\pi^{-1}(D)\right)$ est de dimension 1, alors c'est une droite projective $L \hookrightarrow \mathbb{P}^{q-1}$ et pour tout point $s$ de $D$, on a: $L_{s}=L$. Mais cela est impossible car le morphisme de Gauss $\mathcal{G}$ est fini (lemme 2.9).

Donc $\psi\left(\pi^{-1}(D)\right)$ est une surface et puisque toutes les droites $L_{s}$ (s point de $D$ ) passent par le point $p$, c'est un cône de sommet $p$.

Définition 2.13 Un point $p$ de $\mathbb{P}^{q-1}$ est dit exceptionnel si la fibre $\psi^{-1}(p)$ est de dimension 1 .

Soit $\Delta$ l'ensemble des points exceptionnels. Par la proposition 2.12, chaque point de $\Delta$ est le sommet d'un cône. Etudions la géométrie de $\Delta$ :

Proposition 2.14 L'image de l'application cotangente est de dimension 3 ; $\Delta$ est un fermé de $\mathbb{P}^{q-1}$ vide ou de dimension inférieure ou égale à 1 .

Démonstration Notons $F$ l'image de l'application cotangente et $\psi_{\mid F}$ la restriction de $\psi$ à son image.

Si les fibres générique de $\psi_{\mid F}$ sont de dimension 1 alors $F$ est de dimension 2. En ce cas, pour tout point $p$ de $F$, le schéma $D_{p}$ est de dimension 1 et le fermé $\psi\left(\pi^{-1}\left(D_{p, r e d}\right)\right)$ est de dimension 2 contenu dans la surface irréductible $F$. Cela implique que $\psi\left(\pi^{-1}\left(D_{p, r e d}\right)\right)$ est égal à $F$. Ainsi deux points quelconques de $F$ sont sommets de cônes et sont reliés par une droite $L$ contenue dans $F$ pour laquelle il existe $s$ tel que $L=L_{s}$.

On en déduit que $F$ est un plan projectif. Or la variété $F$ est non-dégénérée dans $\mathbb{P}^{q-1}$, ainsi: $q=3$. Mais on a fait l'hypothèse que la surface $S$ est d'irrégularité $q>3$.

Ainsi, pour un point $p$ générique de l'image de $\psi$, le schéma $D_{p}$ est de dimension nulle et $\psi$ est génériquement finie sur son image.

Le morphisme $\psi$ étant propre et génériquement fini, l'ensemble des points exceptionnels $\Delta$ est un sous-schéma fermé de $\mathbb{P}^{q-1}$ (cf. [8] p. 94). 
2.3 Degré de l'application $\psi$, degré de $\psi_{*} \pi^{*} C$.

\subsubsection{Degré de l'application cotangente et de son image.}

Après avoir étudié les fibres de l'application cotangente, nous étudions son degré.

Soit $F \hookrightarrow \mathbb{P}^{q-1}$ l'image de l'application cotangente, notons deg $F$ son degré et deg $\psi$ le degré de l'application $\psi$ sur son image.

Proposition 2.15 Soit $c_{1}$ et $c_{2}$ les classes de Chern de la surface, alors:

$$
\operatorname{deg} F \operatorname{deg} \psi=c_{1}^{2}[S]-c_{2}[S] .
$$

Démonstration Soit $H$ une section hyperplane de $F$, notons: $h=\psi^{*} H$. Par définition des classes de Chern de $\Omega_{S}$, le cycle $h$ vérifie:

$$
h^{2}-h \cdot \pi^{*} c_{1}\left(\Omega_{S}\right)+\pi^{*} c_{2}\left(\Omega_{S}\right)=0 .
$$

Puisque $c_{1}=-c_{1}\left(\Omega_{S}\right)$ et $c_{2}=c_{2}\left(\Omega_{S}\right)$, on a donc: $h^{3}=-h^{2} \cdot \pi^{*} c_{1}-h \cdot \pi^{*} c_{2}$ et par substitution, on obtient:

$$
h^{3}=h . \pi^{*} c_{1}^{2}+\pi^{*} c_{2} . \pi^{*} c_{1}-h . \pi^{*} c_{2}=h . \pi^{*}\left(c_{1}^{2}-c_{2}\right) .
$$

Le degré du terme de droite est égal à celui de $c_{1}^{2}-c_{2}$. De plus: $\operatorname{deg} h^{3}=\operatorname{deg} H^{3} \operatorname{deg} \psi$ et $\operatorname{deg} H^{3}=\operatorname{deg} F$, donc $\operatorname{deg} F \operatorname{deg} \psi=c_{1}^{2}[S]-c_{2}[S]$ où $c_{1}^{2}[S]$ et $c_{2}[S]$ sont les nombres de Chern de la surface.

Rappelons le diagramme suivant, noté (*):

$$
\begin{array}{ccc}
\mathbb{P}\left(T_{S}\right) & \stackrel{\widetilde{\psi}}{\rightarrow} \mathbb{P}(U) \stackrel{\pi_{1}}{\rightarrow} \mathbb{P}^{q-1} \\
\pi \downarrow & \downarrow \pi_{2} \\
S & \stackrel{\mathcal{G}}{\rightarrow} G(2, q)
\end{array}
$$

Le morphisme $\psi$ vérifie: $\pi_{1} \circ \widetilde{\psi}=\psi$. Notons $\pi_{1}^{\prime}$ la restriction de $\pi_{1}$ à l'image de $\widetilde{\psi}$ sur l'image de $\widetilde{\psi} \circ \pi_{1}$ ainsi:

Proposition 2.16 Le degré de $\psi$ est le produit du degré du morphisme de Gauss $\mathcal{G}$ par le degré de $\pi_{1}^{\prime}$.

Remarque 2.17 L'image de l'application cotangente étant non-dégénérée, son degré est supérieur ou égal à $q-3$, donc le degré de la restriction de l'application cotangente $\psi_{\mid}: \mathbb{P}\left(T_{S}\right) \rightarrow F$ est majoré par $\frac{c_{1}^{2}[S]-c_{2}[S]}{q-3}$.

\subsubsection{Degré du morphisme de Gauss.}

Comme nous allons le voir, on peut créer des surfaces $X$ dont le degré du morphisme de Gauss $\mathcal{G}_{X}$ est arbitraire. Nous saisissons l'occasion pour construire également des surfaces dont le fibré cotangent n'est pas ample:

La donnée d'un revêtement est un triplet $\Lambda=(D, \mathcal{L}, m)$ où $D$ est un diviseur lisse sur $S$, $\mathcal{L}$ un fibré inversible et $m>1$ un entier tel que $\mathcal{L}^{m}=\mathcal{O}_{S}(D)$. Si $D=0$, on demande de plus que $m$ soit le plus petit entier $k>0$ tel que $\mathcal{L}^{k}=\mathcal{O}_{S}$. A une telle donnée est associé un revêtement cyclique $\tau_{\Lambda}: X \rightarrow S$ ramifié en $D^{\prime}=\tau^{-1} D$ où $X=X(\Lambda)$ est une surface lisse. On a : 


\section{Proposition 2.18}

(A) Si la surface $S$ ne possède pas de fibrations, alors il existe une infinité de données de revêtements $\Lambda=(0, \mathcal{L}, m)$ tels que:

(i) la surface $X=X(\Lambda)$ vérifie l'hypothèse 1.3,

(ii) le morphisme $\tau_{\Lambda}^{*}: H^{0}\left(\Omega_{S}\right) \rightarrow H^{0}\left(\Omega_{X}\right)$ est un isomorphisme qui permet d'identifier ces deux espaces.

(iii) sous cette identification, on a: $\mathcal{G}_{X}=\mathcal{G}_{S} \circ \tau_{\Lambda}$. En particulier le degré de $\mathcal{G}_{X}$ est divisible par $m$ et l'application cotangente de X a même image que celle de $S$. Ainsi $\Omega_{X}$ est ample si et seulement si $\Omega_{S}$ est ample.

(B) Si D est ample, alors le fibré cotangent de X n'est pas engendré par ses sections globales au-dessus de $D^{\prime}$ et n'est pas ample.

Démonstration Rappelons ([1], chap. I, Lemma 17.2) que:

$$
\tau_{*} \mathcal{O}_{X}=\oplus_{i=0}^{i=m-1} \mathcal{L}^{-i}
$$

donc $H^{1}\left(X, \mathcal{O}_{X}\right) \simeq \oplus_{i=0}^{i=m-1} H^{1}\left(X, \mathcal{L}^{-i}\right)$.

Supposons $D=0$. Beauville [2] a montré que du fait que $S$ ne possède pas de fibrations, il n'existe qu'un nombre fini de fibrés inversibles $\mathcal{L} \in \operatorname{Pic}^{0}(S)$ tels que $H^{1}(S, \mathcal{L})=0$. Ainsi il existe une infinité de données de revêtements $\Lambda=(0, \mathcal{L}, m)$ tels que $H^{1}\left(S, \mathcal{L}^{-i}\right)=0$ pour $i=1, \ldots, m-1$.

Si $D$ est ample, les groupes $H^{1}\left(S, \mathcal{L}^{-i}\right)$ sont également nuls par le théorème d'annulation de Mumford.

Dans les deux cas, le morphisme injectif $\tau^{*}: H^{0}\left(\Omega_{S}\right) \rightarrow H^{0}\left(\Omega_{X}\right)$ est un isomorphisme. Considérons le diagramme suivant:

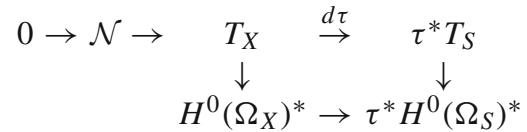

où les flèches verticales sont les morphismes $p r \circ d \vartheta$ définis au paragraphe 2.1 et où $\mathcal{N}$ est le noyau de la différentielle de $\tau$. Le support de $\mathcal{N}$ est le lieu de ramification $D^{\prime}$ et la suite:

$$
0 \rightarrow \mathcal{N} \rightarrow T_{X} \rightarrow H^{0}\left(\Omega_{X}\right)^{*}
$$

est exacte.

Si $D=0$, le corollaire 2.8 implique que l'image d'un point $x$ de $X$ par le morphisme $\mathcal{G}_{X}$ est la même que l'image du point $\tau(x)$ par $\mathcal{G}_{S}$. Ce morphisme $\mathcal{G}_{X}$ se factorise donc par $\tau: X \rightarrow S$ qui est de degré $m$. Les applications cotangentes de $X$ et $S$ ont donc la même image.

Si $D$ est ample, alors $\Omega_{X}$ n'est pas engendré sur $D^{\prime}$. Le théorème 1 de Spurr [17] montre que $\Omega_{X}$ n'est pas ample.

Remarque 2.19 (1) La démonstration de la proposition 2.18 montre que les seuls morphismes $X \rightarrow S$ entre deux surfaces $X, S$ vérifiant l'hypothèse 1.3 et de même irrégularité sont étales et que les applications cotangentes des deux surfaces ont alors la même image.

On ignore si réciproquement, pour une surface $X$ vérifiant l'hypothèse 1.3 et dont le morphisme de Gauss est de degré $m>1$, il existe un morphisme étale $X \rightarrow S$ de degré $m$ dans $S$ de même irrégularité que $X$.

(3) On ne connait pas d'exemples de surfaces ne possédant qu'un nombre fini de courbes non-amples (voir définition supra) $D$ telles que $D^{2}>0$. C'est pour cela que la construction 
d'un revêtement cyclique avec $D>0$ nous a intéressé: la démonstration du théorème 1 de [17] montre que si $\Omega_{S}$ est ample, la courbe $D^{\prime}$ est l'unique obstruction à l'amplitude de $\Omega_{X}$.

\subsubsection{Degré du cycle $\psi_{*} \pi^{*} C$.}

Nous relions ici la géométrie de la surface $S$ à la géométrie de l'image de son application cotangente. La proposition suivante met en rapport les propriétés numériques d'une courbe $C \hookrightarrow S$ aux propriétés numériques du cycle $\psi_{*} \pi^{*} C$ :

Proposition 2.20 Soit $C$ une courbe contenue dans $S$; le degré de $\psi_{*} \pi^{*} C$ est égal à $K C$ où $K$ est un diviseur canonique.

Démonstration Soit $H$ une section hyperplane et $h=\psi^{*} H$. Par définition, le degré de $\psi_{*} \pi^{*} C$ est le degré de l'intersection de $H^{2}$ et de $\psi_{*} \pi^{*} C$. Le morphisme $\psi$ étant propre, on peut utiliser la formule de projection:

$$
\psi_{*}\left(h^{2} \cdot \pi^{*} C\right)=H^{2} \cdot \psi_{*} \pi^{*} C .
$$

Les degrés de $h^{2} \cdot \pi^{*} C$ et de $\psi_{*}\left(h^{2} \cdot \pi^{*} C\right)$ étant égaux, il reste à montrer que le degré de $h^{2} . \pi^{*} C$ est égal à $K C$. Les classes de Chern $c_{1}\left(\Omega_{S}\right), c_{2}\left(\Omega_{S}\right)$ du fibré $\Omega_{S}$ vérifient: $h^{2}=$ $h . \pi^{*} c_{1}\left(\Omega_{S}\right)-\pi^{*} c_{2}\left(\Omega_{S}\right)$ dans l'anneau de Chow de $\mathbb{P}\left(T_{S}\right)$. D'où:

$$
h^{2} . \pi^{*} C=h \cdot \pi^{*} c_{1}\left(\Omega_{S}\right) \cdot \pi^{*} C-\pi^{*} c_{2}\left(\Omega_{S}\right) \cdot \pi^{*} C=h \cdot \pi^{*} c_{1}\left(\Omega_{S}\right) \cdot \pi^{*} C,
$$

or le degré de $c_{1}\left(\Omega_{S}\right) . C$ est $K C$.

\section{Etude des courbes non-amples.}

3.1 Critère de contraction, décomposition du fibré cotangent.

Soit $S$ une surface vérifiant les hypothèses 1.3 et soit $C$ une courbe de $S$ i.e. un schéma de dimension 1 réduit et irréductible. Notons $K$ un diviseur canonique de $S$.

Lemme 3.1 Une courbe $C \hookrightarrow S$ possède une section $t: C \rightarrow \mathbb{P}\left(T_{S}\right)$ contractée en un point par l'application cotangente si et seulement si il existe un morphisme surjectif: $\Omega_{S} \otimes \mathcal{O}_{C} \stackrel{q}{\rightarrow}$ $\mathcal{O}_{C} \rightarrow 0$.

En ce cas le noyau du morphisme q est isomorphe à $\mathcal{O}_{C}(K)$ et la suite exacte:

$$
0 \rightarrow \mathcal{O}_{C}(K) \rightarrow \Omega_{S} \otimes \mathcal{O}_{C} \stackrel{q}{\rightarrow} \mathcal{O}_{C} \rightarrow 0
$$

est scindée.

Démonstration Soit $t: C \rightarrow \mathbb{P}\left(T_{S}\right)$ une section et soit $\Omega_{S} \otimes \mathcal{O}_{C} \rightarrow \mathcal{L} \rightarrow 0$ le quotient correpondant à la section $t$, où le fibré inversible $\mathcal{L}$ vérifie: $t^{*}\left(\mathcal{O}_{\mathbb{P}\left(T_{S}\right)}(1)\right)=\mathcal{L}$ et $(\psi \circ t)^{*}\left(\mathcal{O}_{\mathbb{P} q-1}(1)\right)=\mathcal{L}$.

Le morphisme $\psi \circ t$ est constant si et seulement si $\mathcal{L}$ est trivial. Supposons qu'un tel quotient trivial $q$ existe. Puisque le fibré $\Omega_{S} \otimes \mathcal{O}_{C}$ est engendré par ses sections globales, existe une section $t \in H^{o}\left(C, \Omega_{S} \otimes \mathcal{O}_{C}\right)$ telle que $q(t)$ soit une section non nulle de $\mathcal{O}_{C}$ : cela fournit une section du quotient $q$.

Définition 3.2 Une courbe $C \hookrightarrow S$ est dite non-ample si la restriction du fibré cotangent à $C$ possède un quotient isomorphe à $\mathcal{O}_{C}$. 
3.2 Classification et lissité des courbes non-amples.

Soit $C$ une courbe réduite irréductible contenue dans la surface $S$ et $\Omega_{C}$ le faisceau des différentielles. La suite naturelle suivante:

$$
0 \rightarrow \mathcal{O}_{C}(-C) \rightarrow \Omega_{S} \otimes \mathcal{O}_{C} \rightarrow \Omega_{C} \rightarrow 0
$$

est exacte (cf. [8] proposition 8.12); on l'appellera la suite cotangente de la courbe $C$.

Le théorème suivant classifie partiellement les courbes non-amples $C$ suivant la valeur de l'intersection $C^{2}$ :

Théorème 3.3 Soit $C \hookrightarrow S$ une courbe réduite et irréductible.

(1) La courbe $C$ est une courbe non-ample et vérifie $C^{2}<0$ si et seulement si $C$ est lisse et de genre 1. En ce cas la suite cotangente est scindée.

(2) La courbe $C$ est une courbe non-ample et vérifie $C^{2}=0$ si et seulement si $C$ est lisse, de genre $>1$ et la suite cotangente est scindée. En ce cas le fibré normal $\mathcal{O}_{C}(C)$ est trivial.

La courbe $C$ est lisse si et seulement si le faisceau $\Omega_{C}$ est localement libre de rang 1 ([8] théorème 8.17 , Chap. II). Pour démontrer le théorème 3.3, nous aurons besoin d'un résultat de Lipman qui est la version duale de ce critère de lissité.

Théorème 3.4 (Lipman [10] theorem 1) Une courbe C définie sur un corps de caractéristique nulle est lisse si et seulement si $T_{C}:=\operatorname{Hom}_{\mathcal{O}_{C}}\left(\Omega_{C}, \mathcal{O}_{C}\right)$ est un fibré inversible.

Nous utiliserons également le lemme suivant ([1], lemme 12.2, Chap. II):

Lemme 3.5 Notons $\mathcal{L}$ un fibré inversible de degré négatif ou nul sur une courbe $C$. Le fibré $\mathcal{L}$ est isomorphe au fibré trivial si et seulement si l'espace $H^{o}(C, \mathcal{L})$ est non nul.

Montrons le théorème 3.3: Soit $C$ une courbe contenue dans la surface. La suite duale de la suite exacte:

$$
\mathcal{O}_{C}(-C) \rightarrow \Omega_{S} \otimes \mathcal{O}_{C} \rightarrow \Omega_{C} \rightarrow 0
$$

est la suite exacte:

$$
0 \rightarrow T_{C} \stackrel{i}{\rightarrow} T_{S \mid C} \stackrel{q^{\prime}}{\rightarrow} \mathcal{O}_{C}(C)
$$

Si $C$ est une courbe non-ample, alors la suite exacte:

$$
0 \rightarrow \mathcal{O}_{C}(K) \rightarrow \Omega_{S} \otimes \mathcal{O}_{C} \rightarrow \mathcal{O}_{C} \rightarrow 0
$$

est scindée (proposition 3.1) ; la suite duale est:

$$
0 \rightarrow \mathcal{O}_{C} \stackrel{i^{\prime}}{\rightarrow} T_{S \mid C} \stackrel{q}{\rightarrow} \mathcal{O}_{C}(-K) \rightarrow 0 .
$$


Considérons le diagramme suivant à lignes et colonnes exactes:

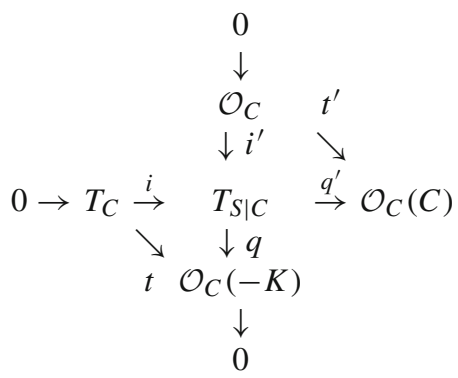

où $t$ et $t^{\prime}$ rendent le diagramme commutatif.

Notation 3.6 Lorsque dans la suite de ce paragraphe $C$ dénote une courbe non-ample de $S$, les notations $i, i^{\prime}, t \ldots$ renvoient à ce diagramme.

Le lemme suivant montre la première affirmation du théorème 3.3 :

Lemme 3.7 Soit $C$ une courbe contenue dans S. Les quatres assertions suivantes sont équivalentes:

(a) la courbe $C$ est non-ample et le morphisme t est nul.

(b) la courbe $C$ est non-ample et le morphisme t' est nul.

(c) la courbe $C$ est une courbe lisse de genre 1.

(d) la courbe $C$ est non-ample et $C^{2}<0$. Si une des assertions est vérifiée, alors la suite cotangente de $C$ est scindée.

Démonstration Soit $C \hookrightarrow S$ une courbe. Supposons que le point (a) soit vérifié i.e. $C$ est non-ample et le morphisme:

$$
t=q \circ i: T_{C} \rightarrow \mathcal{O}_{C}(-K)
$$

est nul. Montrons que (b) est vérifié. Puisque $t=q \circ i$ est nul, par propriété du noyau de $q$, il existe un morphisme $j: T_{C} \rightarrow \mathcal{O}_{C}$ tel que le diagramme suivant:

$$
\begin{array}{cc} 
& \mathcal{O}_{C} \\
j \underset{i}{j} & \downarrow i^{\prime} \\
T_{C} \stackrel{i}{\rightarrow} & T_{S \mid C}
\end{array}
$$

commute et tel que $j$ soit injectif (car $i$ est injectif). Puisque le morphisme $t^{\prime}$ se factorise par $q^{\prime}, t^{\prime}$ est nul sur le sous- $\mathcal{O}_{C}$-module $j\left(T_{C}\right)$ de $\mathcal{O}_{C}$. Le morphisme $t^{\prime}$ se factorise donc par le quotient de $\mathcal{O}_{C}$ par $j\left(T_{C}\right)$. Ce quotient est de torsion et puisque $\mathcal{O}_{C}(C)$ est un fibré inversible, le morphisme:

$$
t^{\prime}: \mathcal{O}_{C} \rightarrow \mathcal{O}_{C}(C)
$$

est nul. Nous avons donc montré que (a) implique (b).

Réciproquement montrons que (b) implique (a). La situation est symétrique de la précédente implication: 
Soit $C$ une courbe non-ample telle que le morphisme $t^{\prime}=q^{\prime} \circ i^{\prime}$ soit nul. Par propriété du noyau de $q^{\prime}$, l'injection $i^{\prime}: \mathcal{O}_{C} \rightarrow T_{S \mid C}$ se factorise par un morphisme $j^{\prime}: \mathcal{O}_{C} \rightarrow T_{C}$ tel que le diagramme suivant:

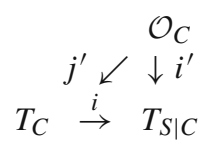

commute. Le morphisme $j^{\prime}$ est de plus injectif car $i^{\prime}$ est injectif. Puisque le morphisme $t$ se factorise par $q, t$ est nul sur le sous- $\mathcal{O}_{C}$-module $j^{\prime}\left(\mathcal{O}_{C}\right)$ de $T_{C}$. Le morphisme $t$ se factorise donc par le quotient de $T_{C}$ par $j^{\prime}\left(\mathcal{O}_{C}\right)$. Ce quotient est de torsion et puisque $\mathcal{O}_{C}(-K)$ est un fibré inversible, le morphisme:

$$
t: T_{C} \rightarrow \mathcal{O}_{C}(-K)
$$

est nul. Nous avons donc montré que (b) implique (a).

Il y a donc équivalence entre les points (a) et (b) et de plus, si $C$ est une courbe non-ample vérifiant (a) et (b), nous avons construit deux morphismes injectifs $j: T_{C} \rightarrow \mathcal{O}_{C}$ et $j^{\prime}: \mathcal{O}_{C} \rightarrow T_{C}$. Le composé $j \circ j^{\prime}: \mathcal{O}_{C} \rightarrow \mathcal{O}_{C}$ est un morphisme injectif, c'est donc un isomorphisme et on en déduit que $j: T_{C} \rightarrow \mathcal{O}_{C}$ est un morphisme surjectif. Le faisceau $T_{C}$ est donc isomorphe à $\mathcal{O}_{C}$. Le théorème de Lipman 3.4 permet alors de conclure que la courbe $C$ est lisse.

En ce cas $\Omega_{C}$ est un fibré inversible, donc:

$$
\operatorname{hom}_{\mathcal{O}_{C}}\left(T_{C}, \mathcal{O}_{C}\right)=\Omega_{C},
$$

et puisque $T_{C}$ est trivial, le fibré $\Omega_{C}$ est trivial. Ainsi $C$ est lisse de genre 1 et nous avons montré que si le point (a) ou (b) est vérifié, alors (c) est vérifié.

Soit $C \hookrightarrow S$ une courbe vérifiant (c) i.e. $C$ est lisse de genre 1. Le quotient naturel $\Omega_{S \mid C} \rightarrow \Omega_{C}$ est un quotient trivial et surjectif donc $C$ est une courbe non-ample. De plus, par adjonction: $C^{2}+K C=0$ et puisque les hypothèses sur $S$ entrainement que le diviseur canonique $K$ est ample, on en déduit que nécessairement $C^{2}$ est strictement négatif. Ceci montre que (c) entraîne (d). (Remarquons de plus que la proposition 3.1 montre que la suite cotangente est scindée).

Soit $C$ une courbe vérifiant l'hypothèse (d) i.e. $C$ est non-ample et $C^{2}<0$. En ce cas, le morphisme $t^{\prime}: \mathcal{O}_{C} \rightarrow \mathcal{O}_{C}(C)$ est une section du fibré $\mathcal{O}_{C}(C)$. Mais ce fibré est de degré $C^{2}$ strictement négatif et le lemme 3.5 montre que $t^{\prime}=0$. Nous avons montré que (d) entraîne $b$ ). Les quatres assertions (a), (b), (c), (d) sont donc équivalentes.

Montrons maintenant la seconde affirmation du théorème 3.3.

- Soit $C \hookrightarrow S$ une courbe non-ample vérifiant $C^{2}=0$, montrons que $C$ est lisse de genre $>1$ et que la suite cotangente est scindée. Le morphisme:

$$
t^{\prime}=q^{\prime} \circ i^{\prime}: \mathcal{O}_{C} \rightarrow \mathcal{O}_{C}(C)
$$

est nécessairement non nul car nous avons montré au lemme 3.7 que $t^{\prime}=0$ entraîne $C^{2}<0$. Ce morphisme $t^{\prime}$ peut être considéré comme une section non nulle de l'espace $H^{o}\left(C, \mathcal{O}_{C}(C)\right)$. Puisque le fibré $\mathcal{O}_{C}(C)$ est de degré $C^{2}=0$, le lemme 3.5 montre que $\mathcal{O}_{C}(C)$ est trivial. Le morphisme:

$$
t^{\prime}=q^{\prime} \circ i^{\prime}: \mathcal{O}_{C} \rightarrow \mathcal{O}_{C}(C) \simeq \mathcal{O}_{C}
$$

est alors un isomorphisme ; le morphisme:

$$
q^{\prime}: T_{S \mid C} \rightarrow \mathcal{O}_{C}(C)=\mathcal{O}_{C}
$$


est donc surjectif et son noyau $T_{C}$ est un fibré inversible. Nous pouvons donc appliquer le théorème de Lipman 3.4 et conclure que si $C$ est une courbe non-ample telle que $C^{2}=0$, alors la courbe $C$ est lisse. De plus, la suite suivante:

$$
0 \rightarrow T_{C} \rightarrow T_{S \mid C} \rightarrow \mathcal{O}_{C}(C)=\mathcal{O}_{C} \rightarrow 0
$$

est exacte et scindée par un multiple du morphisme $i^{\prime}: \mathcal{O}_{C} \rightarrow T_{S \mid C}\left(\right.$ car $t^{\prime}=q^{\prime} \circ i^{\prime}$ est un isomorphisme). La suite cotangente qui est donc scindée.

- Réciproquement, soit $C$ une courbe lisse contenue dans la surface telle que $C^{2}=0$ et telle que la suite cotangente soit scindée ; montrons que $C$ est non-ample. Puisque la suite cotangente est scindée, le fibré $\Omega_{S} \otimes \mathcal{O}_{C}$ est isomorphe à $\mathcal{O}_{C}(-C) \oplus \Omega_{C}$. Mais $\Omega_{S} \otimes \mathcal{O}_{C}$ est engendré par restriction des sections globales de $\Omega_{S}$, donc l'espace $H^{o}\left(C, \mathcal{O}_{C}(-C)\right)$ est non nul. Par le lemme 3.5, $\mathcal{O}_{C}(-C)$ est trivial. Ainsi le fibré $\Omega_{S} \otimes \mathcal{O}_{C} \simeq \mathcal{O}_{C} \oplus \Omega_{C}$ admet un quotient trivial et $C$ est non-ample.

Soit $C$ lisse non-ample vérifiant $C^{2}>0$. La suite cotangente:

$$
0 \rightarrow \mathcal{O}_{C}(-C) \rightarrow \Omega_{S} \otimes \mathcal{O}_{C} \rightarrow \Omega_{C} \rightarrow 0
$$

ne peut être scindée car $\Omega_{S} \otimes \mathcal{O}_{C}$ est engendré par ses sections globales mais le fibré $\mathcal{O}_{C}(-C)$ est de degré $-C^{2}<0$.

Ceci achève la démonstration du théorème 3.3 .

3.3 Exemple des fibrations et géométrie de l'image par $\psi$ de $\pi^{*} C$.

Soit $S$ une surface vérifiant les hypothèses 1.3 et telle qu'il existe un morphisme $f: S \rightarrow B$ surjectif à fibres connexes dans une courbe lisse $B$. En [11] p. 50, Martin-Deschamps montre qu'une fibre lisse $f^{*} b$ ( $b$ point de $B$ ) est non-ample si et seulement si $b$ est un zéro du morphisme de Kodaira-Spencer $\delta$ associé à la fibration (pour la définition de $\delta$ voir [18], exposé III) . La fibration est dite isotriviale si le morphisme de Kodaira-Spencer est nul. Ainsi:

Corollaire 3.8 Quand la fibration est isotriviale, la surface possède une infinité de courbes non-amples.

On dispose ainsi d'exemples de surfaces ayant un nombre infini de courbes non-amples telles que $C^{2}=0$.

Soit $C$ une courbe contenue $S$. Notons $T$ la surface image de $\pi^{*} C$ par $\psi$, notons $\mathcal{K}$ le noyau du morphisme de restriction:

$$
H^{o}\left(\Omega_{S}\right) \rightarrow H^{o}\left(C, \Omega_{S} \otimes \mathcal{O}_{C}\right)
$$

et $k$ la dimension de $\mathcal{K}$. Notons de plus $V$ l'espace quotient de $H^{0}\left(\Omega_{S}\right)$ par $\mathcal{K}$ et $V^{*}$ son dual. L'espace projectif $\mathbb{P}\left(V^{*}\right)$ est naturellement plongé dans $\mathbb{P}\left(H^{0}\left(\Omega_{S}\right)^{*}\right)$.

\section{Proposition 3.9}

(A) L'enveloppe linéaire de la surface $T$ dans $\mathbb{P}^{q-1}$ est l'espace projectif $\mathbb{P}\left(V^{*}\right) \hookrightarrow \mathbb{P}^{q-1}$ de dimension $q-k-1$.

(B) Si $\mathbb{P}\left(V^{*}\right) \neq \mathbb{P}^{q-1}$, alors $C$ vérifie : $C^{2} \leq 0$. Si de plus $C^{2}=0$, alors un multiple de $C$ est une fibre d'une fibration de $S$ dans une courbe de genre $b \geq 1$.

(C) De plus, si $C$ est une courbe non-ample, alors $b=k+1$.

(D) Réciproquement, si C est non-ample et fibre d'une fibration de $S$ dans une courbe de genre $b \geq 1$, alors $b=k+1$. 
Cette proposition entraine qu' une courbe non-ample $C$ telle que $C^{2}>0$ crée une singularité sur l'image de l'application cotangente pourvu que $C^{2}>0$ (voir également corollaire 3.12).

Soit $i: C \hookrightarrow Z$ une courbe sur une surface lisse $Z$. On note $\Omega_{C}$ le faisceau des différentielles de $C$. Pour démontrer la proposition, on utilisera principalement le lemme suivant dû à Spurr ([16] Theorem (1):

Lemme 3.10 Soit $\omega$ une 1-forme holomorphe telle que $i^{*} \omega=0 \in H^{0}\left(C, \Omega_{C}\right)$. Alors la courbe vérifie : $C^{2} \leq 0$. Si de plus $C^{2}=0$, alors un multiple de $C$ est la fibre d'une fibration $f: Z \rightarrow B$ dans une courbe $B$ lisse de genre $b \geq 1$.

Ce lemme a la conséquence directe suivante:

Lemme 3.11 Soit $f: Z \rightarrow B$ une fibration dans une courbe $B$ et soit $i: C \hookrightarrow Z$ une courbe telle que $m C$ soit une fibre de $f$ (pour un certain $m>0$ ). Une 1-forme holomorphe $\tau$ de $Z$ est un élément de $f^{*} H^{0}\left(B, \Omega_{B}\right)$ si et seulement si la restriction $i^{*} \tau \in H^{0}\left(C, \Omega_{C}\right)$ est nulle.

Démonstration (De la proposition 3.9). Posons $Y=\pi^{-1}(C)$ et $j: Y \hookrightarrow \mathbb{P}\left(T_{S}\right)$ le morphisme d'inclusion. Le morphisme $\psi \circ j: Y \rightarrow \mathbb{P}^{q-1}$ est obtenu par le quotient:

$$
H^{o}\left(\Omega_{S}\right) \otimes \mathcal{O}_{Y} \rightarrow j^{*} \mathcal{O}_{\mathbb{P}\left(T_{S}\right)}(1) \rightarrow 0
$$

qui se factorise comme suit:

$$
H^{o}\left(\Omega_{S}\right) \otimes \mathcal{O}_{Y} \rightarrow H^{o}\left(C, \Omega_{S} \otimes \mathcal{O}_{C}\right) \otimes \mathcal{O}_{Y} \rightarrow(j \circ \pi)^{*}\left(\Omega_{S} \otimes \mathcal{O}_{C}\right) \rightarrow j^{*} \mathcal{O}_{\mathbb{P}\left(T_{S}\right)}(1) \rightarrow 0 .
$$

L'image de la surface $Y$ par $\psi$ est donc contenue et non-dégénérée dans $\mathbb{P}\left(V^{*}\right) \hookrightarrow \mathbb{P}^{q-1}$.

Si $\mathbb{P}\left(V^{*}\right)$ est strictement contenu dans $\mathbb{P}^{q-1}$, alors il existe une 1 -forme dont la restriction à $C$ est nulle. Par le lemme 3.10, on a alors: $C^{2} \leq 0$ et de plus, si $C^{2}=0$, alors $C$ est une fibre d'une fibration $f: S \rightarrow B$ dans une courbe de genre $b \geq 1$.

Considérons maintenant $C \hookrightarrow S$ une courbe non-ample. Supposons qu'un multiple $m C$ de $C$ soit la fibre d'une fibration $f: S \rightarrow B$. Par le théorème 3.3, la courbe $C$ est lisse, le fibré normal est trivial et:

$$
\Omega_{S} \otimes \mathcal{O}_{C}=\mathcal{O}_{C} \oplus \mathcal{O}_{C}(K)
$$

où $K$ est un diviseur canonique de $S$. On identifie $\mathcal{O}_{C}(K)$ avec le fibré canonique de $C$. Si $m C=f^{*} p$ (où $p$ est un point de $B$ ), le noyau $\mathcal{K}$ du morphisme de restriction:

$$
H^{o}\left(\Omega_{S}\right) \rightarrow H^{o}\left(C, \Omega_{S} \otimes \mathcal{O}_{C}\right)=H^{0}\left(C, \mathcal{O}_{C}\right) \oplus H^{0}\left(C, \Omega_{C}\right)
$$

est l'image réciproque par $f$ de l'hyperplan de $H^{0}\left(B, \Omega_{B}\right)$ formé des formes nulles en $p$, ainsi $b=k+1$.

Soit $C$ une courbe non-ample de $S$. Soit $T$ le cône image de $\pi^{*} C$ par $\psi$. Si l'image de l'application cotangente est lisse au sommet $t$ du cône $T$, alors les droites passant par $t$ sont contenues dans l'espace projectif tangent à $t$. Ainsi la surface $T$ est contenue dans un sous-espace projectif de dimension 3 de $\mathbb{P}^{q-1}$. Si $q \geq 5$, la proposition 3.9 et le théorème 3.3 impliquent le résultat suivant:

Corollaire 3.12 Sous les hypothèses précédentes, la courbe C vérifie l'une des deux propriétés suivantes:

(a) ou bien $C^{2}<0$ et $C$ est une courbe elliptique,

(b) ou bien $C^{2}=0$ et un multiple de $C$ est la fibre d'une fibration dans une courbe de genre $b$ et $q-3 \leq b \leq q-2$. 
Les courbes de genre petit ont des conséquences particulières sur l'image de l'application cotangente:

Corollaire 3.13 Soit $C \hookrightarrow S$ une courbe lisse de genre 2. La courbe $C$ vérifie: $C^{2} \leq 0$. Si $C^{2}=0$, alors $C$ est une courbe non-ample et il existe un entier $n$ tel que $n C$ soit la fibre d'une fibration $f: S \rightarrow B$ dans une courbe de genre $b=q-3$.

Si de plus $n=1$, alors la fibration est isotriviale à fibres de genre 2 .

Il existe alors une droite $L \hookrightarrow \mathbb{P}^{q-1}$ et une courbe $D \hookrightarrow \mathbb{P}^{q-1}$ disjointes et telles que l'image de l'application cotangente soit balayée par les plans passant par un point de D et contenant $L$.

La dernière assertion est un cas particulier du corollaire 4.10 démontré plus loin.

Démonstration Notons $K$ un diviseur canonique. La restriction à $C$ du morphisme de Gauss $\mathcal{G}: S \rightarrow G(2, q)$ suivis du plongement de Plücker de $G(2, q)$ est donné par le fibré $\mathcal{O}_{C}(K)$. Puisque $\mathcal{G}$ est fini, on a $K C \geq 2$ et si $K C=2$, alors l'image de $C$ par $\mathcal{G}$ est une droite. Une droite de la grassmanienne correspond à l'ensemble des droites passant par un point et contenues dans un plan de $\mathbb{P}^{q-1}$. Ainsi $C$ est non-ample.

L'espace $H^{0}\left(C, \Omega_{S} \otimes \mathcal{O}_{C}\right) \simeq H^{0}\left(C, \mathcal{O}_{C}(K) \oplus \mathcal{O}_{C}\right)$ est de dimension 3, la base de la fibration est donc de genre $q-3$.

Si $n=1$, les fibres lisses sont de genre 2, donc non-amples, et la fibration est isotriviale.

On ne connait pas d'exemple de courbe non-ample d'auto-intersection nulle et qui ne soit pas fibre d'une fibration. La proposition suivante en donne un critère:

Proposition 3.14 Soient $C_{1}, C_{2}$ deux courbes non-amples distinctes contenues dans $S$. Si $C_{1}^{2}=C_{2}^{2}=C_{1} C_{2}=0$, alors il existe une fibration de $S$ telle que des multiples de $C_{1}$ et de $C_{2}$ soient des fibres.

Démonstration Soit $K$ un diviseur canonique de $S$ et $C_{1}, C_{2}$ deux courbes vérifiant l'hypothèse de (a). Il existe deux entiers positifs non nuls $n_{1}$ et $n_{2}$ tels que $n_{1} C_{1} K=n_{2} C_{2} K$. Le diviseur $n_{1} C_{1}-n_{2} C_{2}$ vérifie:

$$
\left(n_{1} C_{1}-n_{2} C_{2}\right)^{2}=\left(n_{1} C_{1}-n_{2} C_{2}\right) K=0 .
$$

Puisque $K^{2}>0$, le théorème de l'indice implique que $n_{1} C_{1}-n_{2} C_{2}$ est algébriquement équivalent à 0 .

Supposons que le fibré $\mathcal{O}_{S}\left(n_{1} C_{1}-n_{2} C_{2}\right)$ soit de torsion: il existe un entier $m>0$ tel que $m n_{1} C_{1}$ soit linéairement équivalent à $m n_{2} C_{2}$. Soit $f$ la fonction rationelle dont le diviseur est $m n_{1} C_{1}-m n_{2} C_{2}$. La fontion $f$ définit un morphisme $f: S \rightarrow \mathbb{P}^{1}$ tel que $m n_{1} C_{1}$ et $m n_{2} C_{2}$ soient des fibres et la proposition est démontrée.

Supposons que $\mathcal{O}_{S}\left(n_{1} C_{1}-n_{2} C_{2}\right)$ ne soit pas de torsion. Soient $\operatorname{Pic}(S)$ la variété de Picard de $S$ et $J\left(C_{1}\right)$ la jacobienne de $C_{1}$. Puisque le fibré normal à $C_{1}$ est trivial (Théorème 3.3) et $C_{1} C_{2}=0$, le fibré inversible $\mathcal{O}_{C_{1}}\left(n_{1} C_{1}-n_{2} C_{2}\right)$ est trivial et le morphisme naturel:

$$
\operatorname{Pic}(S) \rightarrow J\left(C_{1}\right)
$$

possède un noyau de dimension strictement positive. Par dualité, le morphisme:

$$
J\left(C_{1}\right) \rightarrow A
$$

n'est pas surjectif (où $A$ est la variété d'Albanese de $S$ ). Il existe donc une 1-forme holomorphe de $S$ dont la restriction à $C_{1}$ est nulle. Le lemme 3.10 implique alors que $C_{1}$ est le diviseur réduit associé à une fibre d'une fibration. 


\section{Surfaces contenant une infinité de courbes non-amples}

\subsection{Caractérisation de l'image de l'application cotangente}

Rappelons que nous avons noté $\Delta$ le lieu des points exceptionnels i.e. des points $p$ de $\mathbb{P}^{q-1}$ tels que la fibre $\psi^{-1}(p)$ soit de dimension 1 . La dimension de $\Delta$ est inférieure ou égale à 1. Supposons que le fermé $\Delta$ contienne une composante irréductible $B$ de dimension 1 . En ce cas son image inverse $\psi^{-1}(B)$ est de dimension 2. Soit $S^{\prime}$ une composante irréductible de dimension 2 de $\psi^{-1}(B)$ munie de la structure réduite. Notons $\pi^{\prime}$ et $\psi^{\prime}$ les restrictions à $S^{\prime}$ du morphisme de projection $\pi$ et de l'application cotangente:

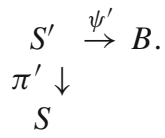

Rappelons qu'une droite de $\mathbb{P}^{q-1}$ est dite sécante d'une courbe $X \hookrightarrow \mathbb{P}^{q-1}$ si elle coupe $X$ en au moins deux points. Si $Y \hookrightarrow \mathbb{P}^{q-1}$ est une seconde courbe, une droite est dite sécante de $X$ et de $Y$ si elle passe par $X$ et $Y$.

Le théorème suivant caractérise les surfaces qui contiennent une infinité de courbes non-amples:

Théorème 4.1 Le morphisme $\pi^{\prime}$ est surjectif. Si s est un point générique de S alors la droite $L_{s}$ coupe $B$ en $d_{o} \in\{1,2\}$ points où $d_{o}$ est le degré de $\pi^{\prime}$.

(1) Si $d_{o}=1$ et si $B$ n'est pas une droite de $\mathbb{P}^{q-1}$, alors le morphisme $\pi^{\prime}: S^{\prime} \rightarrow S$ est un isomorphisme. La surface $S$ possède une fibration isotriviale.

Le lieu des points exceptionnels $\Delta$ est formé de deux courbes lisses irréductibles $B=B_{1}$, $B_{2}$ qui sont images de morphismes canoniques de deux courbes.

L'image de l'application cotangente est la variété des sécantes de ces courbes et l'image du morphisme de Gauss est la surface $B_{1} \times B_{2}$.

(2) Si $d_{o}=2$, alors l'image de l'application cotangente est la variété des sécantes de B. Une courbe non-ample $C \hookrightarrow S$ vérifie: $C^{2}=\operatorname{deg} \mathcal{G}>0$ où $\operatorname{deg} \mathcal{G}$ est le degré du morphisme de Gauss.

L'image du morphisme de Gauss est birationnelle à $B^{(2)}$.

Commençons par le lemme suivant:

Lemme 4.2 Le morphisme $\pi^{\prime}: S^{\prime} \rightarrow$ S est surjectif.

Démonstration Si $S^{\prime}$ est un diviseur vertical pour la projection $\pi$, alors la courbe $B=\psi\left(S^{\prime}\right)$ est une droite projective et pour tout point $s$ de $D=\pi\left(S^{\prime}\right), L_{s}=B$. La courbe $D$ est alors contractée en un point par le morphisme de Gauss. Cela est impossible car $\mathcal{G}$ est fini (lemme 2.9).

Le morphisme $\pi$ étant propre, le morphisme $\pi^{\prime}$ est surjectif.

Le degré $d_{o}$ de $\pi^{\prime}$ est égal au nombre d'intersection dans $\mathbb{P}\left(T_{S}\right)$ de $S^{\prime}$ et de la fibre $\pi^{-1} s$ en un point générique $s$ de $S$.

Lemme 4.3 Soit s un point de la surface. La droite $L_{S}$ coupe B et si s est générique, alors $L_{s}$ coupe la courbe $B$ en $d_{o}$ points. 
Démonstration Par le lemme 4.2, la fibre $\pi^{-1}(s)$ coupe $S^{\prime}$ dans $\mathbb{P}\left(T_{S}\right)$ donc l'image de $\pi^{-1}(s)$ par $\psi$ coupe l'image de $S^{\prime}$ par $\psi$, c'est-à-dire: $L_{s}$ coupe $B$.

L'application cotangente est un plongement sur $\pi^{-1}(s)$. Cela implique que si l'intersection de $\pi^{-1}(s)$ et de $S^{\prime}$ contient $d_{o}$ points distincts, alors $L_{s}$ coupe $B$ en $d_{o}$ points distincts.

Démonstration de la partie (1) du théorème 4.1.

Supposons que le degré $d_{o}$ de $\pi^{\prime}: S^{\prime} \rightarrow S$ vaut 1 .

Lemme 4.4 Si $d_{o}=1$ et si B n'est pas une droite, alors le morphisme $\pi^{\prime}: S^{\prime} \rightarrow S$ est un isomorphisme et la fibration de Stein associée à la fibration $\psi \circ \pi^{\prime-1}: S \rightarrow B$ est isotriviale.

Démonstration Puisque le degré $d_{o}$ de $\pi^{\prime}$ est égal à 1 , le morphisme $\pi^{\prime}: S^{\prime} \rightarrow S$ est birationnel (cf. [12] remarque 6.21). La surface $S$ est normale. Le théorème principal de Zariski montre que si l'application rationnelle $\pi^{\prime-1}$ n'est pas définie en un point $s_{o}$ de $S$, alors l'image inverse $\pi^{\prime-1} s_{o}$ est une courbe de $S^{\prime} \hookrightarrow \mathbb{P}\left(T_{S}\right)$.

En ce cas, la fibre $\pi^{-1} s_{O}$ est contenue dans $S^{\prime}$. Puisque l'application cotangente est un plongement sur $\pi^{-1} s_{o}$, on en déduit alors que $B=\psi^{\prime}\left(S^{\prime}\right)$ est égal à $L_{S_{o}}$. Mais le cas (1) suppose que $B$ n'est pas une droite de $\mathbb{P}^{q-1}$.

Il existe donc un morphisme réciproque $t: S \rightarrow S^{\prime}$ à $\pi^{\prime}$. Les composantes lisses irréductibles d'une fibre de $\psi \circ t: S \rightarrow B$ sont des courbes non-amples. Le corollaire 3.8 permet conclure que la surface $S$ admet une fibration isotriviale.

Il nous reste à caractériser l'image de l'application cotangente. Supposons que la courbe $B$ ne soit pas une droite et que le degré de $\pi^{\prime}: S^{\prime} \rightarrow S$ soit égal à 1 , alors:

Proposition 4.5 Le fermé $\Delta$ est formé de deux courbes lisses irréductibles $C_{1}^{\prime}, C_{2}^{\prime}$ qui sont images de morphismes canoniques de courbes.

L'image de $\psi$ est la réunion des droites sécantes des courbes $C_{1}^{\prime}$ et $C_{2}^{\prime}$.

Pour montrer cette proposition, nous allons nous ramener à supposer que $S$ est un produit de deux courbes.

Soit $S$ une surface vérifiant les hypothèses de la proposition 4.5. Le lemme 4.4 montre que $S$ est isotriviale. Il existe en ce cas un morphisme à fibres connexes $f: S \rightarrow C_{2}^{\prime}$ dont les fibres lisses sont isomorphes entre elles, notons $C_{1}$ une telle fibre, alors:

Lemme 4.6 Il existe une courbe lisse $C_{2}$ et un groupe $G$ agissant algébriquement sur $C_{1}$ et $\mathrm{C}_{2}$ tels que:

(i) La surface $S$ est birationnelle au quotient $\left(C_{1} \times C_{2}\right) / G$.

(ii) La courbe $C_{2}^{\prime}$ est isomorphe à $C_{2} / G$.

(iii) Le diagramme suivant commute:

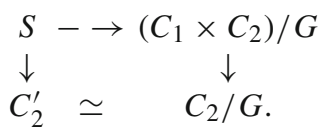

Ici le groupe $G$ agit sur $C_{1} \times C_{2}$ composante par composante (i.e. $\gamma .(a, b)=(\gamma a, \gamma(b))$ et la flèche verticale de droite est la projection naturelle. 
Démonstration Voir [15].

Soit $S$ une surface vérifiant l'hypothèse de la proposition 4.5 et:

$$
C_{1}, C_{2}, G, C_{2}^{\prime}
$$

possédant les propriétés du lemme 4.6.

Puisque la surface $S$ ne contient pas de courbes rationelles, la surface $\left(C_{1} \times C_{2}\right) / G$ est lisse et égale à $S$.

La surface isotriviale $\left(C_{1} \times C_{2}\right) / G$ admet deux fibrations $g: S \rightarrow C_{1}^{\prime}:=C_{1} / G$ et $f: S \rightarrow$ $C_{2}^{\prime}$, notons $\tau: S \rightarrow C_{1}^{\prime} \times C_{2}^{\prime}$ le morphisme $\tau=(g, f)$. Le lemme suivant est la proposition 2.2 de [15]:

Lemme 4.7 L'espace des sections globales du fibré cotangent est:

$$
H^{o}\left(\Omega_{S}\right)=\tau^{*}\left(H^{o}\left(C_{1}^{\prime}, \Omega_{C_{1}^{\prime}}\right) \oplus H^{o}\left(C_{2}^{\prime}, \Omega_{C_{2}^{\prime}}\right)\right) .
$$

Les surfaces $S$ et $C^{\prime}{ }_{1} \times C_{2}^{\prime}$ ont la même irrégularité. Par la remarque 2.19, on obtient:

Corollaire 4.8 Par l'identification $H^{o}\left(S, \Omega_{S}\right) \simeq H^{o}\left(C_{1}^{\prime} \times C_{2}^{\prime}, \Omega_{C_{1}^{\prime} \times C_{2}^{\prime}}\right)$, les applications cotangentes de $S$ et du produit $C_{1}^{\prime} \times C_{2}^{\prime}$ ont la même image.

Pour terminer la démonstration de la proposition 4.5, il nous reste à comprendre quelle est l'image de l'application cotangente d'une surface isotriviale $S=C_{1} \times C_{2} / G$ quand $G=\{1\}$ est le groupe trivial. Puisque $S$ est de type général, ces deux courbes sont de genres respectifs $g_{1}, g_{2}$ supérieurs ou égaux à 2. le lemme suivant est classique:

Lemme 4.9 Soit $C_{1}, C_{2}$ deux courbes de genre $g_{1}>1$ et $g_{2}>1$. La surface $C_{1} \times C_{2}$ vérifie l'hypothèse 1.3 et ses nombres de Chern vérifient:

$$
\begin{aligned}
& c_{1}^{2}[S]=8\left(g_{1}-1\right)\left(g_{2}-1\right) \\
& c_{2}[S]=4\left(g_{1}-1\right)\left(g_{2}-1\right)
\end{aligned}
$$

Soit donc $S=C_{1} \times C_{2}$ avec $C_{1}, C_{2}$ de genre $g_{1}>1$ et $g_{2}>1$. Notons $\pi_{i}: S \rightarrow C_{i}, i \in\{1,2\}$ les projections respectives. Le fibré cotangent vérifie:

$$
\Omega_{S}=\pi_{1}^{*} \Omega_{C_{1}} \oplus \pi_{2}^{*} \Omega_{C_{2}},
$$

et l'espace des sections globales $H^{o}\left(\Omega_{S}\right)$ s'identifie à $H^{o}\left(C, \Omega_{C_{1}}\right) \oplus H^{o}\left(C_{2}, \Omega_{C_{2}}\right)$. La surface $S=C_{1} \times C_{2}$ est d'irrégularité $g_{1}+g_{2}>3$.

Pour $i \in\{1,2\}$, notons $\phi_{i}: C_{i} \rightarrow \mathbb{P}^{g_{1}+g_{2}-1}$ le composé du morphisme canonique:

$$
C_{i} \rightarrow \mathbb{P}\left(H^{o}\left(C_{i}, \Omega_{C_{i}}\right)^{*}\right)
$$

avec le plongement naturel:

$$
\mathbb{P}\left(H^{o}\left(C_{i}, \Omega_{C_{i}}\right)^{*}\right) \hookrightarrow \mathbb{P}\left(H^{o}\left(C_{1}, \Omega_{C_{1}}\right)^{*} \oplus H^{o}\left(C_{2}, \Omega_{C_{2}}\right)^{*}\right)=\mathbb{P}^{g_{1}+g_{2}-1} .
$$

Notons de plus $C_{i}^{\prime}$ l'image du morphisme $\phi_{i}$. La proposition suivante caractérise l'image de l'application cotangente de $S$ et est une conséquence directe de la définition du morphisme de Gauss 2.7:

Proposition 4.10 Soit $s=\left(p_{1}, p_{2}\right)$ un point de la surface $S=C_{1} \times C_{2}$. La droite $L_{s}$ passe par les points $\phi_{1}\left(p_{1}\right)$ et $\phi_{2}\left(p_{2}\right)$; les courbes lisses $C_{1}^{\prime}$ et $C_{2}^{\prime}$ forment l'ensemble des points exceptionnels.

La première partie du théorème 4.5 est donc démontrée. 
Démonstration de la partie (2) du théorème 4.1.

Supposons maintenant que le degré $d_{o}$ du morphisme $\pi^{\prime}: S^{\prime} \rightarrow S$ soit supérieur ou égal à 2 .

Soit $s$ un point générique de $S$. Par le lemme 4.3, la droite $L_{s}$ passe par $d_{o}>1$ points de $B$ et il y a trois possibilités:

(i) Il existe un point $b_{1}$ de $B$ telle que la droite générique $L_{s}$ passe par $b_{1}$.

(ii) Il existe des points $b_{1}, \neq b_{2}$ de $B$ telle que la droite $L_{s}$ passe par $b_{o}, b_{1}$.

(iii) L'intersection de $L_{s}$ et de $B$ se fait en deux points variables de $B$.

Le cas i) est exclu car alors $\psi$ aurait une fibre de dimension 2 en $b_{1}$, ce qui contredirait le lemme 2.11. Le cas ii) est exclu car l'image de l'application cotangente serait alors une droite.

Ceci montre que l'image de $\psi$ est la variété développée par les sécantes de $B$. Puisque l'image de $\psi$ est non-dégénérée, la courbe $B$ est non-dégénérée dans $\mathbb{P}^{q-1}$. Les fibres de $\pi^{\prime}$ sont donc finies car si $S^{\prime}$ contenait une fibre $\pi^{-1} s_{o}$, alors $B$ serait une droite et l'image de l'application cotangente serait cette droite.

Soient $b, b^{\prime}$ deux points génériques de $B$. La courbe $C_{b}=\pi\left(\psi^{-1}(b)\right)$ est une courbe non-ample et $C_{b}^{2}=C_{b} C_{b^{\prime}}$. Une sécante de $B$ générique est repérée de manière unique par les deux points $b, b^{\prime}$ de l'intersection de $L$ et $B$, et le cardinal des droites $L_{s}$ telles que $L_{s}=L$ est égal à deg $\mathcal{G}$ (voir les diagrammes du paragraphe 2.3.1), ainsi: $C_{b}^{2}=\operatorname{deg} \mathcal{G}>0$.

Ceci termine la démonstration du théorème 4.1

4.2 Compléments au cas d'une surface produit de deux courbes.

Soit $C_{1}, C_{2}$ deux courbes de genres respectifs $g_{1}, g_{2}$ supérieur ou égaux à 2 et soit $n \in\{0,1,2\}$ le nombre de courbes hyperelliptiques parmis $C_{1}, C_{2}$.

Proposition 4.11 La surface $S=C_{1} \times C_{2}$ vérifie l'hypothèse 1.3. Le degré de l'image $F$ de l'application cotangente de la surface $C_{1} \times C_{2}$ est:

$$
2^{2-n}\left(g_{1}-1\right)\left(g_{2}-1\right) \text {. }
$$

et le degré de l'application cotangente et du morphisme de Gauss est $2^{n}$.

Démonstration On reprend les notations de la démonstration du théorème 4.1. Soit $i \in\{1,2\}$, notons $k_{i}$ le degré de $C^{\prime}{ }_{i} \hookrightarrow \mathbb{P}^{q-1}$. Si la courbe $C_{i}$ est hyperelliptique alors $k_{i}=g_{i}-1$, sinon $k_{i}=2\left(g_{i}-1\right)$.

Soit $\left(\omega_{1}, \omega_{2}\right) \in H^{o}\left(C_{1}, \Omega_{C_{1}}\right) \times H^{o}\left(C_{2}, \Omega_{C_{2}}\right)$ deux formes génériques. L'intersection de $F$ avec l'hyperplan $H_{1}=\left\{\omega_{1}=0\right\}$ est une surface formée des $k_{1}$ cônes reliant $k_{1}$ points de $C_{1}^{\prime}$ aux points de $C_{2}^{\prime}$.

L'intersection de $H_{1} F$ avec l'hyperplan $H_{2}=\left\{\omega_{2}=0\right\}$ est formée des droites reliant $k_{1}$ points de $C_{1}$ à $k_{2}$ points de $C_{2}$ et est de degré $k_{1} k_{2}$. Ainsi deg $F=k_{1} k_{2}$.

Pour le degré de l'application cotangente, on utilise la proposition 2.15.

4.3 Exemple du produit symétrique.

Soit $C$ une courbe de genre $g>3$. L'involution $\tau:\left(P_{1}, P_{2}\right) \rightarrow\left(P_{2}, P_{1}\right)$ agit sur la surface $C \times C$. On notera $S=C^{(2)}$ la surface quotient et

$$
\begin{gathered}
\eta: C \times C \rightarrow C^{(2)} \\
\left(P_{1}, P_{2}\right) \rightarrow P_{1}+P_{2}
\end{gathered}
$$


le morphisme quotient. Il existe un isomorphisme naturel:

$$
H^{o}\left(C, \Omega_{C}\right) \rightarrow H^{o}\left(S, \Omega_{S}\right)
$$

appelé morphisme trace [6] qui permet d'identifier les deux espaces $H^{o}\left(\Omega_{S}\right)$ et $H^{o}\left(C, \Omega_{C}\right)$. Puisque la courbe $C$ n'est pas hyperelliptique, le morphisme canonique:

$$
\phi: C \rightarrow \mathbb{P}^{g-1}=\mathbb{P}\left(H^{o}\left(\Omega_{S}\right)^{*}\right)
$$

est un plongement.

Proposition 4.12 Soit $C$ une courbe non-hyperelliptique de genre $g>3$.

La surface $S=C^{(2)}$ vérifie l'hypothèse 1.3.

Pour tout point $P$ de $C$, la courbe $P+C \hookrightarrow C^{(2)}$ est une courbe non-ample et vérifie $(P+C)^{2}=1$.

L'image de l'application cotangente est la variété des sécantes de $C \hookrightarrow \mathbb{P}\left(H^{o}\left(C, \Omega_{C}\right)^{*}\right)=$ $\mathbb{P}\left(H^{o}\left(\Omega_{S}\right)^{*}\right)$ et la courbe $C \hookrightarrow \mathbb{P}^{g-1}$ est le lieu des points exceptionnels.

Si $g \geq 5$, on a:

$$
\operatorname{deg} \psi=1, \operatorname{deg} F=2(g-1)(g-3),
$$

où $\operatorname{deg} \psi$ est le degré de l'application cotangente et $\operatorname{deg} F$ celui de son image. Si $g=4$, alors deg $\psi=6$.

Rappelons que pour un point $s$ de $S$, nous avons noté $L_{s}$ l'image par l'application $\psi$ de $\pi^{-1}(s)$. Pour un point $s$ de $S$, on note $\mathcal{I}_{s}$ l'idéal de définition de $s$. Pour un diviseur $D$ de $C$, on note $\Omega_{C}(D)$ le fibré $\Omega_{C} \otimes \mathcal{O}_{C}(D)$.

Lemme 4.13 Supposons que C soit non-hyperelliptique de genre supérieur ou égal à 4.

(a) Le morphisme naturel $\vartheta: C^{(2)} \rightarrow J(C)$ est un morphisme d'Albanese et un plongement. Le fibré cotangent $\Omega_{S}$ est engendré par ses sections globales.

(b) Les invariants numériques de $S=C^{(2)}$ sont:

$$
c_{1}^{2}[S]=(g-1)(4 g-9), c_{2}[S]=(g-1)(2 g-3), q=g .
$$

(c) Soit $s=P_{1}+P_{2}$ un point de $S$, l'image par l'isomorphisme trace de l'espace $H^{o}$ $\left(C, \Omega_{C}\left(-P_{1}-P_{2}\right)\right)$ est $H^{o}\left(S, \mathcal{I}_{S} \Omega_{S}\right)$.

(d) Soit $s=P_{1}+P_{2} \in C^{(2)}$ avec $P_{1} \neq P_{2}$. La droite $L_{s}$ est la droite passant par les points $\phi\left(P_{1}\right)$ et $\phi\left(P_{2}\right)$.

Démonstration Le point (a) utilise le fait que $C$ n'est pas hyperelliptique et l'appendice [13]. Le point (b) est classique. Le point (c) résulte de [6]. Par le corollaire 2.8, le point (d) est une autre formulation de (c).

Le degré de la variété des sécantes à une courbe lisse de genre $g$ et de degré $d$ dans un espace projectif de dimension $\geq 4$ est égal a

$$
-g+(d-1)(d-2) / 2
$$

donc le degré de l'image de $\psi$ est $2(g-1)(g-3)$ si $g \geq 5$. Par la proposition 2.15, on a $\operatorname{deg} \psi \operatorname{deg} F=2(g-1)(g-3) \operatorname{donc} \operatorname{deg} \psi=1$.

Soit $P \in C$, pour tout élément $s$ de $P+C$, la droite $L_{s}$ (correspondant au point $\mathcal{G}(s)$ ) passe par $\phi(P)$. Le point $\phi(P)$ est donc sommet d'un cône et $P+C$ est une courbe non-ample. 
On vérifie que $(P+C)^{2}=1$.

La proposition 4.12 est donc démontrée.

Les revêtements étales de surfaces isogènes à un produit donnent des exemples différents de surfaces possédant une infinité de courbes non-amples telles que $C^{2}>0$.

\section{References}

1. Barth, W., Hulek, K., Peters, C., Van De Ven, A.: Compact complex surfaces, Ergeb. Math. Grenzgeb. vol. 4, seconde édition augementée, Springer (2004)

2. Beauville, A.: Annulation du H1 et systèmes paracanoniques sur les surfaces. J. Reine Angew. Math. 388, 149-157 (1988)

3. Conduche, D., Palmeiri, E.: On the Chern ratio for surfaces with ample cotangent bundle. Mathematische (Catania) 61(1), 143-156 (2006)

4. Debarre, O.: Varieties with ample cotangent bundle. Compo. Math. 141(6), 1445-1459 (2005)

5. Gieseker, D.: On a theorem of Bogomolov on Chern classes of stables bundle. Am. J. Math. 101, 7785 (1979)

6. Griffiths, P.: Variations on a theorem of Abel. Inv. Math. 35, 321-390 (1976)

7. Griffiths, P., Harris, J.: Principes of Algebraic Geometry. Wiley (1978)

8. Hartshorne, R.: Algebraic Geometry. Springer G.T.M. 52 (1977)

9. Hartshorne, R.: Ample vector bundles. Publi. Math. I.H.E.S. 319-350 (1966)

10. Lipman, J.: Free derivation modules on algebraic varieties. Am. J. Math. t.87, 874-898 (1965)

11. Martin-Deschamps, M.: Propriétés de descente des variétés à fibré cotangent ample. Ann. Inst. Fourier 33, 39-64 (1984)

12. Milne, J.: Lectures on algebraic geometry. www.math.sa.umich.edu/jmilne/

13. Mumford, D.: The Red Book of Varieties and Schemes. L.N.M. 1358, Springer (1999)

14. Ran, Z.: The structure of Gauss-like maps. Comp. Math. 52, 171-177 (1984)

15. Serrano, F.: Isotrivial fibred surfaces. Annali di Math. Pura et Appli., 171 (1996)

16. Spurr, M.: On the zero set of a holomorphic one-form on a compact complex manifold. Trans. Am. Soc. 308, 329-339 (1988)

17. Spurr, M.: Branched coverings of surfaces with ample cotangent bundle. Pac. J. Math. 164, 129-146 (1994)

18. Szpiro, L. (éditeur).: Séminaire sur les pinceaux de courbes de genre au moins deux. Astérisque $86, \mathrm{SMF}$ (1986) 